\title{
GAMETERAPIA NA COORDENAÇÃO MOTORA E INTEGRAÇÃO VISO-MOTORA EM PESSOAS COM SÍNDROME DE DOWN
}

Maysa Venturoso Gongora Buckeridge Serra

Universidade de Franca.

E-mail: ma_buckeridge@hotmail.com

Cynthia Yukiko Hiraga

Universidade Estadual Paulista Júlio de Mesquita Filho.

\section{Danielle de Freitas Gonçalves}

Universidade de Franca.

\section{Letícia Hellen Pereira Silva}

Universidade de Franca

\section{Carolina Moreira Martins}

Universidade de Franca.

Maria Georgina Marques Tonello

Universidade de Franca
RESUMO: O objetivo deste estudo foi examinar os efeitos da gameterapia na coordenação motora e integração viso-motora em indivíduos com síndrome de Down (SD). Quatro indivíduos com SD participaram do estudo. Os testes de Coordenação Corporal para Crianças (Körperkoordinationstest Für Kinder - KTK) e o Teste do Desenvolvimento da Integração Viso-Motora (Test of Visual-Motor Integration - VMI) foram aplicados. Os participantes realizaram 12 sessões de gameterapia, com duração de 20 minutos cada, com jogos do console Nintendo Wii. Os participantes foram submetidos aos testes KTK e VMI antes e após o programa de gameterapia. Todos os participantes apresentaram aumento no escore do teste KTK e no teste $V M I$, dois participantes apresentaram aumento do escore final nas duas partes que compóem o teste. Os resultados sugerem que a gameterapia altera variáveis relativas a coordenação motora e componentes que envolvem a percepção em indivíduos com SD.

PALAVRAS-CHAVE: Jogos de Vídeo; Deficiência Intelectual; Exercício.

\section{GAME TERAPY IN MOTOR COORDINATION AND VISO- MOTOR INTEGRATION IN PERSONS WITH DOWN SYNDROME}

ABSTRACT: The purpose of this study was to assess the effects of game therapy in the motor coordination and visual-motor integration in individuals with Down syndrome (DS). Four people with DS participated in the study. The Test of Body Coordination for Children (Körperkoordinationstest Für Kinder - KTK) and the Test of VisualMotor Integration (VMI) were applied. The participants performed 12 sessions of game therapy, lasting 20 minutes each, with games of the Nintendo Wii console. The participants did KTK and VMI tests before and after the game therapy program. All participants improved their scores in the KTK and VMI tests, two participants increased the final score in the two parts of the test. The results suggest that game therapy changes variables related to motor coordination and components involving perception in individuals with DS.

KEY WORDS: Video Games; Intellectual Disability; Exercise.

\section{INTRODUÇÃO}

Pessoas com síndrome de Down (SD) apresentam exagerada fraqueza muscular das articulações e hipotonia. Outra característica encontrada com frequência é a disfunção no controle postural, 
relacionada com dificuldades de coordenação motora, problemas com integração sensório-motora ou movimentos de modo geral (POLASTRI; BARELA, 2005; CARVALHO; MOREIRA; PEREIRA, 2010). Em especial, pessoas com SD praticam menos exercícios físicos na recreação do que a comunidade em geral, sendo que esta prática pode contribuir para a prevenção de problemas de saúde, sejam de natureza física (por exemplo, aumento do índice de massa corporal - IMC) mental ou psicossocial (SOTORIVA; SEGURA, 2013; LANTE et al., 2014).

De acordo com a literatura, crianças com SD apresentam baixa força muscular e esta força não melhora com a maturação (SILVA et al, 2017). Outros autores (ROSSATO et al., 2017) sugerem que a redução da força muscular em SD, na fase adulta, tende a limitar seu desempenho nas atividades de vida diária e na vida laboral. A força muscular, especialmente a dos membros inferiores, em indivíduos com SD é fundamental para a saúde geral, melhoria da produtividade profissional e para desenvolver a independência nas atividades de vida diária (RIMMER et al., 2010). A participação de pessoas com SD em programas de atividade física vem sendo cada vez mais estimulada. No entanto, ainda há desafios para consolidar estratégias de ensino e terapêuticas que considerem as diferenças dessas pessoas de modo a colaborar na promoção de seu desenvolvimento cognitivo, psicossocial e motor (RODRIGUES; LIMA, 2014).

Tendo em vista uma possível dependência dos outros, e no contexto das circunstâncias sociais em que os indivíduos com SD vivem, a atividade física tem baixa prioridade em suas vidas, sendo necessários novos métodos para estimular essa população. Tecnologias produzidas pela indústria do entretenimento, tais como vídeo games, podem ser utilizadas como ferramenta de trabalho dos profissionais da saúde e da educação (SCHIAVINATO et al., 2011). Pessoas com deficiência intelectual, tais como as com SD, podem maximizar a aquisição de competências motoras e cognitivas a partir de diferentes fontes de informação sensorial.

Nesse contexto, a realidade virtual (RV) oferece um ambiente gerado por computador em três dimensões (3D) que pode ser utilizado para treinamento $\mathrm{e}$, consequentemente, fazer com que a pessoa com SD produza conhecimento para melhorar sua qualidade de vida (HALL; CONBOY-HILL; TAYLOR 2011). O uso da RV como uma ferramenta de trabalho de profissionais da saúde e da educação ganhou grande destaque nos últimos anos, principalmente com o uso do Nintendo Wii e do X-Box 360, promovendo terapias baseadas em jogos de vídeo game, ou seja, a gameterapia (SCHIAVINATO et al., 2011; SERRA et al., 2016).

Indivíduos com SD podem beneficiar-se do uso de vídeo game como complemento às demais terapias. O tratamento baseado na gameterapia pode ser individualizado, além de proporcionar motivação extra e feedback em tempo real, de modo a auxiliar na melhora da qualidade de vida (ROCHA; DEFAVARI; BRANDÃO, 2012). Há um crescente interesse na aplicabilidade da gameterapia em pessoas com SD. O presente estudo teve por objetivo examinar os efeitos de um programa de gameterapia na coordenação motora e integração visomotora de indivíduos com síndrome de Down (SD).

Consideramos este estudo importante, pois pessoas com SD podem sentir-se motivadas a praticar gameterapia por ser esta uma atividade física realizada em um ambiente de realidade virtual, diferente das atividades físicas comuns que os profissionais de saúde oferecem a essa população. Assim, a gameterapia é capaz de melhorar alguns aspectos da vida de pessoas com SD, como força muscular, flexibilidade, independência e sua qualidade de vida (SCHIAVINATO et al., 2011).

\section{METODOLOGIA}

Em 2016, foram selecionados quatro indivíduos com SD, alunos regulares de uma escola especial para pessoas com deficiência intelectual em um município do interior do estado de São Paulo. Mais especificamente, dois do sexo masculino e dois do sexo feminino, com idades entre 17 e 36 anos, que participaram voluntariamente do estudo. Os critérios de inclusão no estudo restringiam o mesmo a indivíduos com SD que apresentassem pelo menos $75 \%$ de frequência nas sessões de gameterapia, aptos fisicamente, ou seja, não apresentassem incapacidade física para interagir com os jogos do console Nintendo Wii. Como fator de exclusão, foram 
considerados os alunos que apresentassem deficiências físicas que impediam ou limitavam a movimentação na interação com os jogos do console Nintendo Wii. Para tratamento e análise dos dados, os participantes foram identificados por número, de modo a manter a privacidade de cada um. Este estudo foi aprovado pelo Comitê de Ética em Pesquisa da Universidade de Franca (Parecer $\mathrm{n}^{\mathrm{O}}$ 1.619.722).

As medidas antropométricas [peso, estatura, índice de massa corporal (IMC), circunferência abdominal (CA), circunferência da cintura (CC), circunferência do quadril (CQ)], dos participantes foram coletadas e registradas antes do início e após da gameterapia. A partir das medidas de circunferência da cintura e quadril, a relação cintura/quadril (RCQ) foi calculada. As coletas foram realizadas em ambiente fechado, amplo, livre de obstáculos e interferências visuais, com piso liso e boa iluminação.

Para avaliar as competências motoras relacionadas ao equilíbrio dinâmico e à coordenação motora, foi utilizada a bateria de testes de Coordenação Corporal para Crianças (Körperkoordinationstest Für Kinder - KTK), criada por Kiphard e Schilling em 1974. A bateria é composta de quatro tarefas: equilíbrio na trave, saltos monopodais, saltos laterais e transferência lateral. Em todas as tarefas é exigida coordenação de padrões motores básicos: equilíbrio, ritmo, lateralidade, velocidade e agilidade. Para registro e controle do avaliador, foi preparada uma ficha de avaliação.

Para avaliar a integração viso-motora foi aplicado o teste de Integração Viso-Motora (Beery VMI) (BEERY; BEERY, 2010), O teste VMI referente à integração viso-motora apresenta uma sequência de 24 formas geométricas, em um formulário próprio, para serem reproduzidas/copiadas pelo avaliado usando lápis e papel em um espaço específico do formulário. O Beery $V M I$ inclui dois outros testes adicionais: percepção visual (PV) e coordenação motora fina (CMF). O teste referente à percepção visual é composto por 27 figuras modelo. Baseado nessas figuras, o avaliado deve visualizar e assinalar (ou apontar) dentre um conjunto de opçóes qual das opções apresentadas assemelha-se ao modelo. A parte do teste que examina a coordenação motora fina consiste de testes como tracejar linhas entre pontos preestabelecidos no formulário. A complexidade desses testes aumenta a cada figura completada.

Após a primeira aplicação dos testes KTK e Beery VMI nos participantes (pré-intervenção), estes realizaram 12 sessões de atividades físicas em ambiente virtual (ou seja, gameterapia), com duração de 20 minutos cada, com o videogame Nintendo Wii. No presente estudo foram utilizados Cds do Wii Play, Wii Sports e Wii Sports Resort que requerem habilidades como equilíbrio, coordenação motora geral, percepção visual, e integração viso-motora dos participantes. Para projetar o conteúdo dos CDs em tela, foi utilizado um aparelho de televisão. As sessões foram realizadas três vezes por semana até atingir um total de 12 sessões.

Após o programa de gameterapia, os dados antropométricos dos participantes e os dados das baterias de testes KTKe Beery VMI foram novamente coletados (ou seja, pós-intervenção). Os dados coletados das baterias de testes KTK e Beery VMI foram registrados inicialmente em folha de avaliação que acompanha as baterias, conforme manual de instruções (KIPHARD; SCHILLING, 1974; BEERY; BEERY, 2010). Posteriormente, esses dados foram organizados em planilha eletrônica Microsoft Office Excel 2010. Os dados foram apresentados em relação à pontuação padrão dos testes KTK e Beery VMI.

\section{RESULTADOS}

Os resultados das medidas antropométricas são apresentados na Tabela 1. Os resultados encontrados não mostraram alterações no IMC, entretanto, houve uma redução da RCQ em dois participantes do sexo feminino. 
Tabela 1. Resultados das medidas antropométricas em pré-intervenção e pós-intervenção dos participantes

\begin{tabular}{cccccccccc}
\hline \multicolumn{1}{c}{ Pré-intervenção } \\
\hline Part. & Sexo & Idade (anos) & Peso $(\mathrm{kg})$ & Altura $(\mathrm{m})$ & IMC & CA (cm) & CC (cm) & CQ (cm) & RCQ \\
\hline 1 & Homem & 36 & 68 & 1.56 & 27.94 & 93 & 85 & 100 & 0.85 \\
2 & Homem & 17 & 88.8 & 1.68 & 31.46 & 102 & 90 & 116 & 0.78 \\
3 & Mulher & 22 & 48.8 & 1.44 & 23.53 & 80.5 & 69 & 95 & 0.73 \\
4 & Mulher & 22 & 67.7 & 1.47 & 31.14 & 98 & 90 & 105 & 0.86 \\
\hline & & & & Pós-intervenção & & & \\
\hline Part. & Sexo & Idade (anos) & Peso (kg) & Altura (m) & IMC & CA (cm) & CC (cm) & CQ (cm) & RCQ \\
\hline 1 & Homem & 36 & 68 & 1.56 & 27.90 & 92 & 85.5 & 101 & 0.85 \\
2 & Homem & 17 & 88.2 & 1.68 & 31.25 & 107 & 91 & 119 & 0.76 \\
3 & Mulher & 22 & 48 & 1.44 & 23.15 & 80.5 & 68 & 99 & 0.69 \\
4 & Mulher & 22 & 65.1 & 1.47 & 30.13 & 99 & 87 & 107 & 0.81 \\
\hline
\end{tabular}

Nota: Part.: Participantes; kg: Quilogramas; M: Metros; IMC: Índice de Massa Corporal; CM: Centímetros; CA: Circunferência abdominal; CC: Circunferência da Cintura; CQ: Circunferência do Quadril; RCQ: Relação Cintura/Quadril.

Os resultados da bateria de testes KTK mostraram ao quociente motor total (QMT), três dos participantes que todos os participantes apresentaram melhoras no apresentaram melhoras nos resultados finais. Os quociente motor (QM) 2 e QM4 no KTK. Com relação resultados são apresentados na Tabela 2.

Tabela 2. Resultado do teste KTK em pré-intervenção e pós-intervenção dos participantes

\begin{tabular}{cccccc}
\hline & \multicolumn{2}{c}{ Pré-intervenção } & & \\
\hline Part. & QM1 & QM2 & QM3 & QMT & \\
1 & 43 & 16 & 45 & 38 & 142 \\
2 & 42 & 25 & 35 & 32 & 134 \\
3 & 35 & 20 & 26 & 36 & 117 \\
4 & 37 & 14 & 23 & 34 \\
\hline \\
\hline
\end{tabular}

Nota: Part.: Participantes; QM: Quociente Motor; QMT: Quociente Motor Total.

Os resultados do teste Beery VMI são divididos após a gameterapia, tornando-se, assim, classificáveis em três partes, como apresentado na Tabela 3. Dois pelo teste Beery VMI.

participantes mostraram aumento da pontuação padrão 
Tabela 3. Resultado do teste Beery VMI, PV e CMF em pré-intervenção e pós-intervenção dos participantes

\begin{tabular}{|c|c|c|c|c|c|c|c|c|c|c|c|c|}
\hline & \multicolumn{4}{|c|}{ VMI } & \multicolumn{4}{|c|}{ PV } & \multicolumn{4}{|c|}{ CMF } \\
\hline & \multicolumn{2}{|c|}{ Pontuação } & \multicolumn{2}{|c|}{ Percentil } & \multicolumn{2}{|c|}{ Pontuação } & \multicolumn{2}{|c|}{ Percentil } & \multicolumn{2}{|c|}{ Pontuação } & \multicolumn{2}{|c|}{ Percentil } \\
\hline & Pré & Pós & Pré & Pós & Pré & Pós & Pré & Pós & Pré & Pós & Pré & Pós \\
\hline Part. 1 & 15 & 15 & - & - & 15 & 16 & - & - & 15 & 13 & - & - \\
\hline Part. 2 & 5 & 4 & - & - & 16 & 18 & - & 0.02 & 19 & 20 & 0.09 & 0.6 \\
\hline Part. 3 & 15 & 4 & - & - & 14 & 14 & - & - & 12 & 11 & - & - \\
\hline Part. 4 & 15 & 14 & - & - & 15 & 17 & - & 0.02 & 15 & 16 & - & - \\
\hline
\end{tabular}

Nota: Pré: Pré-intervenção; Part.: Participantes; Pós: Pós-intervenção; -: Não classificável.

\section{DISCUSSÃO}

Gameterapia é uma abordagem recente que muitos profissionais da saúde estão utilizando para finalidades como a melhoria das variáveis cognitivas, equilíbrio e coordenação motora (SCHIAVINATO et al., 2010; SERRA et al., 2016). Seus benefícios, ainda que positivos, não são conclusivos. No presente estudo verificou-se que após um programa de gameterapia, dois participantes apresentaram diminuição da relação cintura/quadril. Sabe-se que pessoas com SD apresentam anormalidades determinadas por fatores genéticos, como hipotonia muscular, e a prevalência de sobrepeso e obesidade é maior nesta população comparada à população em geral sem síndrome (SILVA; SANTOS; MARTINS, 2006).

Alguns estudos sugerem que a hipotonia muscular, presente nesta população, pode afetar a prática de exercícios físicos, sendo que a prática traz benefícios para a saúde, como redução do risco cardiovascular, um dos principais riscos que pessoas com SD apresentam (IZQUIERDO-GOMEZ et al., 2014; ESPOSITO et al, 2012; MATUTE-LLORENTE et al., 2013). A falta de envolvimento em atividades físicas pelos indivíduos com SD pode ocorrer por falta de estimulação, e a utilização de tecnologia para a prática de atividades físicas pode promover a motivação e aderência à prática dessa população. A gameterapia mostra-se eficiente por ser realizada em ambiente virtual tridimensional estimulante, capaz de promover a prática regular de exercícios físicos para pessoas com SD (SINGH et al, 2013).

Este estudo mostrou que, em geral, todos os participantes apresentaram melhorias na coordenação motora após o programa de gameterapia. Pessoas com baixa coordenação motora podem não se sentir motivadas a praticar exercícios físicos (CAIRNEY et al, 2017). A gameterapia pode ser aplicada em um ambiente fechado na qual o jogador pode sentir-se desinibido para realizar as tarefas do jogo e oferece um feedback instantâneo que pode contribuir para o aprendizado e melhora da coordenação motora e equilíbrio (LAUBER; KELLER, 2014). Além disso, este instrumento mantém a atenção e o envolvimento do jogador por oferecer um ambiente tridimensional diferente e estimulante (STRAUDI et al, 2017).

A coordenação motora e o equilíbrio são essenciais para a aquisição de habilidades motoras adequadas e, consequentemente, uma pessoa com boas habilidades motoras sente-se mais engajada a participar de atividades físicas, a realizar tarefas da escola e a procurar atividades de lazer (CAIRNEY et al, 2017). Com o treino da coordenação motora e do equilíbrio, a pessoa com SD sentirá confiança para demonstrar suas habilidades motoras quando forem requisitadas por alguma tarefa, seja no dia-a-dia ou em alguma atividade física (STRAKER et al, 2011).

Este estudo demonstrou que os participantes tinham integração viso-motora abaixo do considerado adequado pelo teste Beery VMI para a idade de cada participante (BEERY; BEERY 2010). No entanto, após a gameterapia dois participantes aumentaram sua pontuação no desempenho do teste de percepção visual e foram classificáveis no teste. Esses resultados mostraram que é possível melhorar aspectos relacionados a integração viso-motora, ou seja, percepção visual, em pessoas com SD através de um programa de gameterapia, pois os 
jogos oferecidos pelo vídeo game têm uma interface visual gráfica na qual o usuário precisa continuamente identificar estímulos visuais relevantes para interagir no contexto virtual do jogo (STRAUDI et al, 2017).

A integração viso-motora tem sido descrita como multifacetada e influenciada por fatores como funções visuais receptivas, funções cognitivas visuais, capacidade motora fina e integração de processos visuais, cognitivos e motores (DANKERT; DAVIES; GAVIN, 2003). As limitações cognitivas das pessoas com SD têm uma influência direta na realização de tarefas no dia-a-dia que requerem a integração viso-motora preservada (DI BLASI et al, 2007). Nesse sentido, a gameterapia oferece jogos capazes de treinar funções importantes para melhorar a integração viso-motora do usuário (HALL; CONBOY-HILL; TAYLOR, 2011), sendo que pessoas com SD precisam priorizar esse treinamento.

Neste estudo foi utilizado o vídeo game Nintendo Wii, que tem um controle que permite a movimentação ativa do jogador. Assim, o jogador é capaz de treinar habilidades como coordenação motora, equilíbrio e funções responsáveis para manter a integração visomotora (ROCHA; DEFAVARI; BRANDÃO,2012). Nesses últimos anos, a gameterapia começou a ser usada como ferramenta de treinamento por ser de baixo custo, alta portabilidade e capacidade de envolver o jogador (LAVER et al, 2015). O uso do vídeo game, tal como o console do Nintendo Wii, como terapia principal ou adjunta para melhorar habilidades motoras e cognitivas que traz benefícios ainda é uma incógnita a ser abordada em pesquisas futuras.

\section{5}

\section{CONCLUSÃO}

A gameterapia aumentou a coordenação motora e a integração viso-motora em pessoas com SD. Esse estudo foi realizado com uma amostra de quatro participantes, no entanto, os dados mostram-se fundamentais, pois revelam o potencial que um programa de gameterapia pode oferecer à pessoas com SD por tratar-se de uma população que precisa de estímulos diferentes para manter uma vida saudável.

REFERÊNCIAS
BEERY, K. E.; BEERY, N. A. The Beery-Buktenica Developmental Test of Visual-Motor Integration. 6.ed. Texas: Pearson, 2010.

CAIRNEY, J.; VELDHUIZEN, S.; KING-DOWLING, S.; FAUGHT, B. E.; HAY, J. Tracking cardiorespiratory fitness and physical activity in children with and without motor coordination problems. Journal of Science and Medicine in Sport, v. 20, n. 4, p. 380-385, 2017.

CARVALHO, R. L.; MOREIRA, T. M.; PEREIRA, M. A. G. Shantala no desenvolvimento neuropsicomotor em portador se Síndrome de Down. Revista Científica da UNIFAE, v. 4, n. 1, p. 1, 2010.

DANKERT, H. L.; DAVIES, P. L.; GAVIN, W. J. Occupational therapy effects on visual-motor skills in preschool children. American Journal of Occupational Therapy, v. 57, n. 5, p. 542-549, 2003.

DI BLASI, F. D.; ELIA, F.; BUONO, S.; RAMAKERS, G. J.; DI NUOVO, S. F. Relationships between visual-motor and cognitive abilities in intellectual disabilities. Perceptual and Motor Skills, v. 104, n. 3, p. 763-772, 2007.

ESPOSITO, P. E.; MACDONALD, M.; HORNYAK, J. E.; ULRICH, D. A. Physical activity patterns of youth with Down syndrome. Intellectual and Developmental Disabilities, v. 50, n. 2, p. 109-119, 2012.

HALL, V.; CONBOY-HILL, S.; TAYLOR, D. Using virtual reality to provide health care information to people with intellectual disabilities: acceptability, usability, and potencial utility. Journal of Medical Internet Research, v. 13, n. 4, 2011.

IZQUIERDO-GOMEZ, R.; MARTÍNEZ-GÓMEZ, D.; ACHA, A.; VEIGA, O. L.; VILLAGRA, A.; DIAZ-CUETO, M. Objective assessment of sedentary time and physical activity throughout the week in adolescents with Down syndrome. The UP\&DOWN study. Research in Developmental Disabilities, v. 35, n. 2, p. 482-489, 2014.

KIPHARD, E. J.; SCHILLING, V. F. Köper-koordinationstest für kinder: KTK. Beltz Test Gmbh, Weinhein, 1974.

LANTE, K.; STANCLIFFE, R. J.; BAUMAN, A.; PLOEG, H. 
P.; JAN, S.; DAVIS, G. M. Embedding sustainable physical activities into the everyday lives of adults with intellectual disabilities: a randomised controlled Trial. BioMed Central Public Health, v. 14, 2014.

LAUBER, B.; KELLER, M. Improving motor performance: selected aspects of augmented feedback in exercise and health. European Journal of Sport Sciences, v. 14, p. 36-43, 2014.

LAVER, K. E.; GEORGE, S.; THOMAS, S.; DEUTSCH, J. E.; CROTTY, M. Virtual reality for stroke rehabilitation. The Cochrane Database Systematic Reviews, v. 12, n. 2, 2015.

MATUTE-LLORENTE， A.; GONZÁLEZ-AGÜERO， A.; GÓMEZ-CABELLO, A.; VICENTE-RODRÍGUEZ， G.; CASAJÚS, J. A. Physical activity and cardiorespiratory fitness in adolescents with Down syndrome. Nutrición Hospitalaria, v. 28, n. 4, p. 1151-1155, 2013.

POLASTRI, P. F.; BARELA, J. A. Perception- action coupling in infants with Down Syndrome: effects of experience and practice. Adapted Physical Activity Quartely, v. 1, n. 22, p. 39-58, 2005.

RIMMER, J. H.; YAMAKI, K.; LOWRY, B. M.; WANG, E.; VOGEL, L. C. Obesity and obesity-related secondary conditions in adolescents with intellectual/developmental disabilities. Journal of Intellectual Disability Research, v. 54, n. 9, p. 787-794, 2010.

ROCHA, P.; DEFAVARI, A. H.; BRANDÃO, P. S. Estudo da viabilidade da utilização do Kinect como ferramenta no atendimento fisioterapêutico de pacientes neurológicos. XI SBGames, v. 2, n 4, p. 16-22, 2012.

RODRIGUES, M. N.; LIMA, S. R. Atividades motoras aquáticas na coordenação corporal de adolescentes com deficiência intelectual. Revista Brasileira de Ciências do Esporte, v. 36, n.2, p. 369-381, 2014.

ROSSATO, M.; DELLAGRANA, R. A.; DE SOUZA BEZERRA, E.; DA COSTA, R. M.; DOS SANTOS, J. O.; SILVA, D. A.; DIEFENTHAELER, F. Comparison of body adiposity index (BAI) and air displacement plethysmograph with estimations of \% body fat in adults with Down's syndrome.

\section{European Journal of Clinical Nutrion, 2017.}

SINGH, D. K. A.; MOHD NORDIN, N. A.; ABD AZIZ, N. A.; LIM, B. K.; SOH, L. C. Effects of substituting a portion of standard physiotherapy time with virtual reality games among community-dwelling stroke survivors. BioMed Central Neurology, v. 13, n. 199, 2013.

SCHIAVINATO, A. M.; MACHADO, B. C.; PIRES, M. A.; BALDAN, C. Influência da realidade virtual no equilíbrio de paciente portador de Disfunção Cerebelar - estudo de caso. Revista Neurociências, v. 19, n. 1, p. 119-127, 2011.

SCHIAVINATO, A. M, BALDAN, C., MELATTO, M., LIMA, L. S. Influência do Wii Fit no equilíbrio de paciente com disfunção cerebelar: estudo de caso. Journal of the Health Sciences Institute; v. 28, n. 1, p. 50-52, 2010.

SERRA, M. V. G. B.; HIRAGA, C. Y.; VASSIMON, H. S.; QUEMELO, P. R. V.; TONELLO, M. G. M. Game therapy as therapeutic practice for disabled persons. The FIEP Bulletin, v. 86, p. 631-634, 2016.

SILVA, D. L.; SANTOS, J. A. R.; MARTINS, C. F. Avaliação da composição corporal em adultos com síndrome de Down. Arquivos de Medicina, v. 20, n. 4, p. 103-110, 2006.

SILVA, V.; CAMPOS, C.; SÁ, A.; CAVADAS, M.; PINTO, J.; SIMÕES, P.; MACHADO, S.; MURILLO-RODRÍGUEZ, E.; BARBOSA-ROCHA, N. Wii-based exercise program to improve physical fitness, motor proficiency and functional mobility in adults with Down syndrome. Journal of Intellectual disability research, 2017.

SOTORIVA, P.; SEGURA, D. C. A. Aplicação do método bobath no desenvolvimento motor de crianças portadoras de síndrome de down. Saúde e Pesquisa, v. 6, n. 2, p. 323-330, 2013.

STRAKER, L. M.; CAMPBELL, A. C.; JENSEN, L. M.; METCALF, D. R.; SMITH, A. J.; ABBOTT, R. A.; POLLOCK, C. M.; PIEK, J. P. Rationale, design and methods for a randomised and controlled Trial of the impacto $f$ virtual reality games on motor competence, physical activity, and mental health in children with 
developmental coordination disorder. BioMed Central

Public Health, v. 18, n. 11, 2011.

STRAUDI, S.; SEVERINE, G. CHARABATI, A. S.; PAVARELLI, C.; GAMBERINI, G.; SCOTTI, A.; BASAGLIA, N. The effects of video game therapy on balance and attention in chronic ambulatory traumatic brain injury: an exploratory study.

BioMed Central Neurol, v. 17, n. 86, 2017.

Recebido em: 30 de maio de 2017 Aceito em: 30 de agosto de 2017 